\title{
The Diabetes Drug Liraglutide Prevents Degenerative Processes in a Mouse Model of Alzheimer's Disease
}

\author{
Paula L. McClean, Vadivel Parthsarathy, Emilie Faivre, and Christian Hölscher \\ School of Biomedical Sciences, Ulster University, Coleraine BT52 1SA, Northern Ireland, United Kingdom
}

\begin{abstract}
Type 2 diabetes is a risk factor for Alzheimer's disease, most likely linked to an impairment of insulin signaling in the brain. The incretin hormone glucagon-like peptide-1 (GLP-1) facilitates insulin signaling, and novel long-lasting GLP-1 analogs, such as liraglutide, are on the market as diabetes therapeutics. GLP-1 has been shown to have neuroprotective properties in vitro and in vivo. Here we tested the effects of peripherally injected liraglutide in an Alzheimer mouse model, $\mathrm{APP}_{\text {swe }} / \mathrm{PS} 1_{\Delta \mathrm{E} 9}(\mathrm{APP} / \mathrm{PS} 1)$. Liraglutide was shown to cross the blood-brain barrier in an acute study. Liraglutide was injected for 8 weeks at $25 \mathrm{nmol} / \mathrm{kg}$ body weight i.p. once daily in 7-month-old APP/PS1 and wild-type littermate controls. In APP/PS1 mice, liraglutide prevented memory impairments in object recognition and water maze tasks, and prevented synapse loss and deterioration of synaptic plasticity in the hippocampus, commonly observed in this model. Overall $\beta$-amyloid plaque count in the cortex and dense-core plaque numbers were reduced by $40-50 \%$, while levels of soluble amyloid oligomers were reduced by $25 \%$. The inflammation response as measured by activated microglia numbers was halved in liraglutidetreated APP/PS1 mice. Numbers of young neurons in the dentate gyrus were increased in APP/PS1 mice with treatment. Liraglutide treatment had little effect on littermate control mice, whose behavior was comparable to wild-type saline controls; however, synaptic plasticity was enhanced in the drug group. Our results show that liraglutide prevents key neurodegenerative developments found in Alzheimer's disease, suggesting that GLP-1 analogs represent a novel treatment strategy for Alzheimer's disease.
\end{abstract}

\section{Introduction}

Type 2 diabetes mellitus (T2DM) has been identified as a risk factor for Alzheimer's disease (AD) (Hoyer, 2004; Hölscher, 2005; Craft, 2007; Perry et al., 2007). Insulin receptors are desensitized in the brains of $\mathrm{AD}$ patients (Craft, 2007). The impairment of insulin signaling in the brain could play a role in the development of neurodegenerative disorders, as it leaves neurons more exposed to damaging influences (Hoyer, 2004; Hölscher, 2005; Craft, 2007).

Glucagon-like peptide-1 (GLP-1) is an endogenous 30 aa incretin hormone (Lovshin and Drucker, 2009). Currently, the GLP-1 receptor agonists exendin-4 (Exenatide, Byetta) and liraglutide (Victoza) are approved for treatment of T2DM (Lovshin and Drucker, 2009). These analogs are injected subcutaneously and are well tolerated. Incretins do not affect blood glucose levels in normoglycemic people and can be given to nondiabetic patients (Vella et al., 2002).

GLP-1 also acts as a growth factor in the brain, and has been shown to induce neurite outgrowth and to protect against oxidative injury in cultured neuronal cells (Perry et al., 2007).

Received Jan. 31, 2011; revised March 14, 2011; accepted March 21, 2011.

Author contributions: C.H. designed research;P.L.M., V.P., and E.F. performed research;P.L.M. and V.P. analyzed data; C.H. wrote the paper.

This project was funded by the Alzheimer's Society (Grant 97) with support from The Henry Smith Charity. We thank Prof. P. Flatt and Dr. V. A. Gault of the Ulster University diabetes research group for advice and support.

C.H. declares competing commercial interests. He is a named inventor on a patent held by the University of Ulster for the use of GLP-1 analogs as treatments of neurodegenerative diseases.

Correspondence should be addressed to Dr. Christian Hölscher, School of Biomedical Sciences, Ulster University, Cromore Road, Coleraine BT52 1SA, Northern Ireland, UK. E-mail: c.holscher@ulster.ac.uk.

DOI:10.1523/JNEUROSCI.0529-11.2011

Copyright $\odot 2011$ the authors $\quad 0270-6474 / 11 / 316587-08 \$ 15.00 / 0$
Furthermore, mice that overexpress GLP-1 receptors in the hippocampus (a brain area that is involved in memory formation) showed increased neurite growth and improved learning (During et al., 2003). The deletion of the GLP-1 receptor impairs learning of new tasks and of developing long-term potentiation (LTP) of synaptic transmission in the hippocampus (Abbas et al., 2009). Moreover, GLP- 1 and exendin- 4 have been shown to reduce endogenous levels of $\beta$-amyloid (A $\beta$ ) in the brain (Perry et al., 2003). GLP-1 analogs induce the proliferation of neuronal progenitor cells in the brains of mice (During et al., 2003). Increasing cell proliferation has the potential to facilitate the repair of neuronal networks in cortical tissue and could have beneficial effects in patients with $\mathrm{AD}$ (Greenberg and Jin, 2006). We have previously shown that injection of $\mathrm{Val}(8) \mathrm{GLP}-1$ intraperitoneally for 3 weeks rescued LTP in the hippocampus of an $\mathrm{APP}_{\text {swe }} / \mathrm{PS}_{\triangle \mathrm{E} 9}$ (APP/PS1) mouse model of AD (Gengler et al., 2010b).

An important aspect of developing new drug treatments for neurodegenerative diseases of the brain is the permeability of the novel compounds across the blood-brain barrier (BBB). Several studies have shown that the GLP-1 and GLP-1 analogs such as Val(8)GLP-1 cross the BBB (Kastin et al., 2002; Gengler et al., 2010b). Peripheral injection of GLP-1 analogs intraperitoneally increased neuronal progenitor proliferation in the brain (During et al., 2003) and enhanced LTP and paired-pulse facilitation (PPF) in the hippocampus (Gengler et al., 2010a). These results suggest that treatment with GLP-1 analogs beneficially affects a number of the therapeutic targets associated with $\mathrm{AD}$, such as impaired cognitive performance, reduced neuronal communication, neurodegenerative processes, and reduced neuronal regeneration (Hölscher and Li, 2010). Here we show that liraglutide 
crosses the BBB, and show the effect of 8 weeks of liraglutide intraperitoneal administration in an $\mathrm{APP}_{\text {swe }} / \mathrm{PS}_{\Delta \mathrm{E} 9}$ mouse model of AD (Jankowsky et al., 2001). We analyzed impairments in memory formation, synaptic plasticity of the hippocampus, synaptic density, levels of potentially neurotoxic soluble oligomers in the brain (Townsend et al., 2006), total brain amyloid precursor protein (APP) levels, the formation of amyloid plaques, and the inflammation response in the cortex, as well as the formation of new neurons as induced by liraglutide.

\section{Materials and Methods}

\section{Animals}

$\mathrm{APP}_{\text {swe }} / \mathrm{PS}_{\triangle \mathrm{Eg}}$ mice with a C57BL/6 background were obtained from The Jackson Laboratory (http://research.jax.org/repository/alzheimers. html). Heterozygous males were bred with wild-type C57BL/6 females bought locally (Harlan). Offspring were ear punched and genotyped using PCR with primers specific for the APP-sequence (forward: GAATTCCGACATGACTCAGG, reverse: GTTCTGCTGCATCTTGGACA). For details, see Gengler et al. (2010b). Mice not expressing the transgene were used as wild-type controls. Male animals were used in all studies. Animals were caged individually and maintained on a $12 \mathrm{~h}$ light/ dark cycle (lights on at 8:00 A.M., off at 8:00 P.M.), in a temperature-controlled room $\left(21.5 \pm 1{ }^{\circ} \mathrm{C}\right)$. Food and water were available ad libitum. Animals were handled daily for 2 weeks before commencement of the study.

Mice were 7 months of age when treatment began. A baseline object recognition task was conducted on days 1 and 2 of injection to determine acute effects of intraperitoneal liraglutide [ $25 \mathrm{~nm} / \mathrm{kg}$ body weight (bw)] or saline $(0.9 \% \mathrm{w} / \mathrm{v})$ injection. Mice were then injected daily with liraglutide $(25 \mathrm{~nm} / \mathrm{kg} \mathrm{bw})$ or saline $(0.9 \% \mathrm{w} / \mathrm{v})$ for 8 weeks before further behavioral tasks were conducted ( $n=12$ for each group). All experiments were licensed by the UK Home Office in accordance with the Animals (Scientific Procedures) Act of 1986.

\section{Peptides}

Liraglutide was purchased from GL Biochem (Shanghai) Ltd. The purity of the peptide was analyzed by reversed-phase HPLC and characterized using matrix-assisted laser desorption/ionization time of flight (MALDI-TOF) mass spectrometry.

\section{Object recognition task}

The object recognition task was conducted in a gray-colored aluminum open-field arena (58 $\mathrm{cm}$ in diameter; $31-\mathrm{cm}$-high wall). The arena was illuminated, from $2 \mathrm{~m}$ above, using a $60 \mathrm{~W}$ lamp. Objects for exploration were red cubes ( $1.8 \mathrm{~cm}$ diameter) and white balls $(2.6 \mathrm{~cm}$ diameter). The arena and objects were cleaned with $70 \%$ ethanol between trials to prevent the buildup of olfactory cues.

Mice received a session of $5 \mathrm{~min}$ in the empty open-field arena, $24 \mathrm{~h}$ before exposure to objects, to habituate them to the apparatus and test room. Motor activity was recorded by total path, number of lines crossed, and speed. The number of rearing events (forepaws elevated from the floor) was considered to be an index of exploratory behavior. The number of grooming sessions and the number of fecal pellets are common measures of anxiety in mice.

Twenty-four hours after habituation, each mouse was subjected to a 10 min acquisition trial, during which they were placed in the open-field arena in presence of two identical objects (cube or ball) situated at $15 \mathrm{~cm}$ from the arena wall. After a $3 \mathrm{~h}$ retention interval, the mice were placed 0.01 ; all groups $n=12$ ).

\section{Wild type mice}
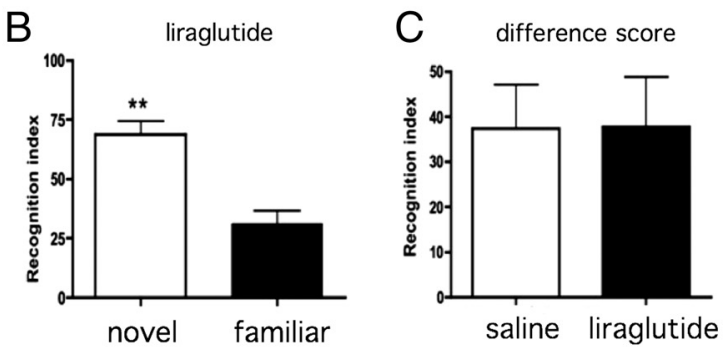

APP/PS1 mice
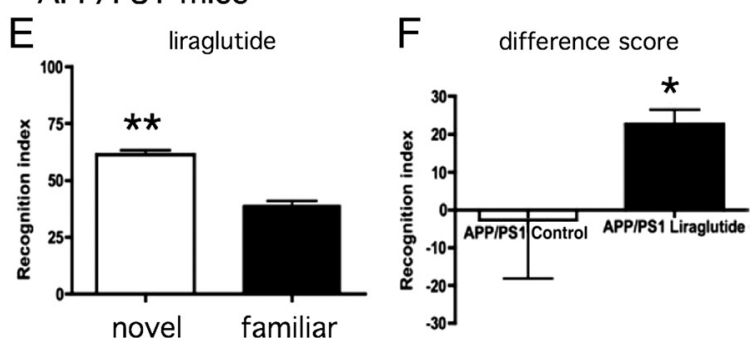

Figure 1. Effect of saline and liraglutide treatment on recognition memory in an object recognition task. In the object recog( Overall difference scores were significantly increased in APP/PS1 liraglutide-treated mice $(\boldsymbol{F})$ (Student's $t$ test, ${ }^{*} p<0.05,{ }^{* *} p<$

back into the arena and exposed to the familiar object and to a novel object for a further $10 \mathrm{~min}$. Object location and novel and familiar objects were randomized throughout the trial.

Total time spent exploring each of the two objects (when the animal's snout was directly toward the object at a distance $\leq 2 \mathrm{~cm}$ ) was recorded. Recognition index was defined as the amount of time exploring the familiar object or the novel object over the total time spent exploring both objects multiplied by 100 , and was used to measure recognition memory $(\mathrm{TA} \text { or } \mathrm{TB} /(\mathrm{TA}+\mathrm{TB}))^{*} 100$, where A represents familiar object and $\mathrm{B}$, novel object ( $n=12$ per group).

\section{Morris water maze task}

The maze was made of white opaque plastic with a diameter of $120 \mathrm{~cm}$ and $40-\mathrm{cm}$-high walls, and was filled with water at $25^{\circ} \mathrm{C}$ to avoid hypothermia. A small escape platform $(10 \times 6.5 \times 21.5 \mathrm{~cm})$ was placed at a fixed position in the center of one quadrant, $25 \mathrm{~cm}$ from the perimeter, and was hidden $1 \mathrm{~cm}$ beneath the water surface. The room contained a number of fixed visual cues on the walls.

Acquisition phase. The acquisition trial phase consisted of 4 training days (day 1-4) and four trials per day with a $15 \mathrm{~min}$ intertrial interval. Four points equally spaced along the circumference of the pool (north, south, east, west) served as the starting position, which was randomized across the four trials each day. If an animal did not reach the platform within $90 \mathrm{~s}$, it was guided to the platform, where it had to remain for $30 \mathrm{~s}$, before being returned to its home cage. Mice were kept dry, between trials, in a plastic holding cage filled with paper towels. The path length and escape latencies were recorded ( $n=12$ per group).

Probe trial. One day after finishing the acquisition task (day 5), a probe trial was performed to assess the spatial memory (after a $24 \mathrm{~h}$ delay). The platform was removed from the maze and animals were allowed to swim freely for $60 \mathrm{~s}$. Spatial acuity was expressed as the amount of time spent in the exact area where the escape platform was located.

\section{Reversal Morris water maze task}

One day after completion of the Morris water maze task, a reversal training program was commenced. This involved changing the location of the 


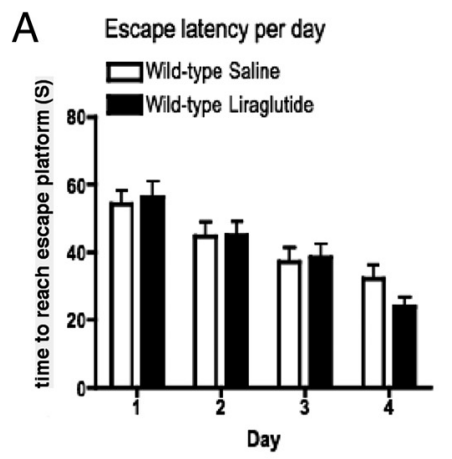

B Probe trial

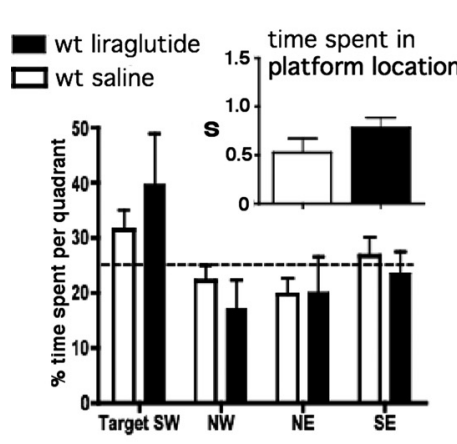

C Reversal escape latency per day

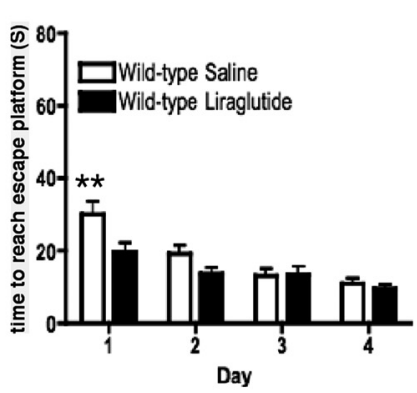

D Reversal probe trial

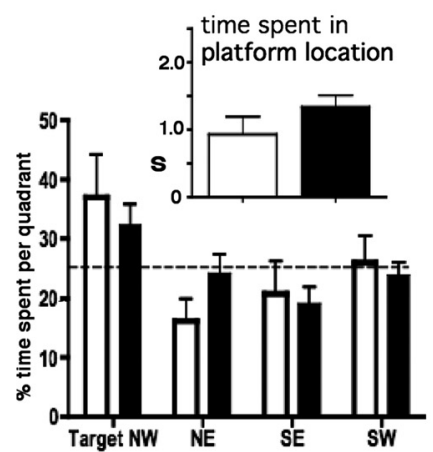

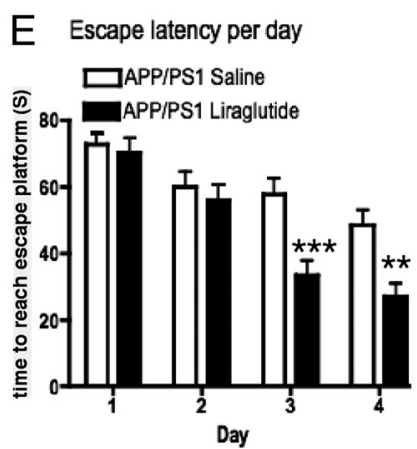

F Probe trial

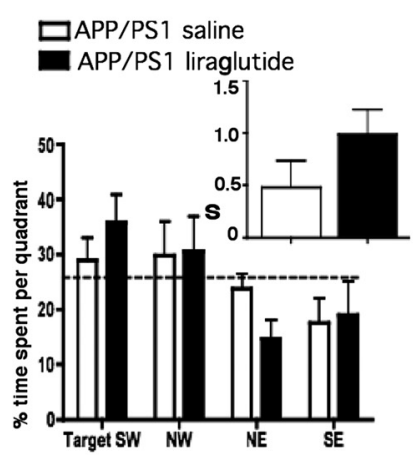

G Reversal escape latency per day

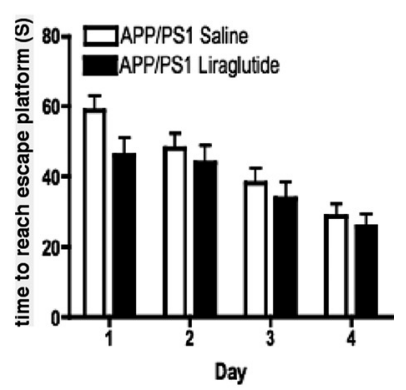

$\mathrm{H}$ Reversal probe trial

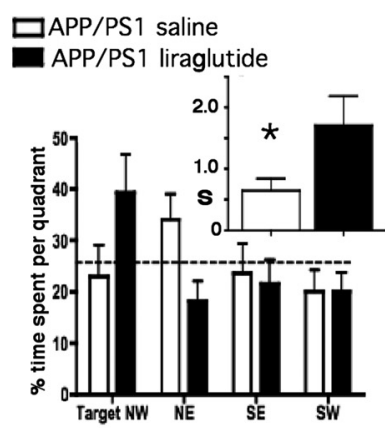

Figure 2. Liraglutide-treated APP/PS1 mice demonstrate improved memory in Morris water maze learning task. After $56 \mathrm{~d}$ treatment with $0.9 \%$ saline or liraglutide ( $25 \mathrm{~nm} / \mathrm{kg}$ bw), mice were exposed to a Morris water maze learning paradigm involving $4 \mathrm{~d}$ acquisition, followed by a probe trial. Mice then received $4 \mathrm{~d}$ reversal training (with platform location moved) followed by a reversal probe trial. No appreciable differences were observed in the learning behavior of wild-type mice in normal $(\boldsymbol{A}, \boldsymbol{B})$ and reversal $(\boldsymbol{C}, \boldsymbol{D})$ water maze training and probe trials, despite a reduced escape latency on day 1 of reversal training $(\boldsymbol{C})$. Liraglutide-treated APP/PS1 group learned the task faster in the acquisition phase, on days 3 and 4 (E), and remembered the location of the platform after the reversal probe trial $(\boldsymbol{F}$, inset), showed no difference in the reversal acquisition phase ( $\boldsymbol{G})$, but showed good memory in the probe task $(\boldsymbol{H}$, inset) (Student's $t$ test, $p=0.0465)$, while the saline APP/PS1 group did not. ${ }^{*} p<0.05,{ }^{* *} p<0.01,{ }^{* * *} p<0.001$, all groups $n=12$.

escape platform and a $4 \mathrm{~d}$ acquisition phase, followed by a probe trial on day 5 (as outlined above; $n=12$ per group).

\section{Analysis of transport across the $B B B$}

Female wild-type (C57BL/ 6 background) mice were used, 5 per group. Mice were injected intraperitoneally with $2.5,25$, or $250 \mathrm{nmol} / \mathrm{kg}$ bw liraglutide or saline $(0.9 \% \mathrm{w} / \mathrm{v})$ as control. At $30 \mathrm{~min}$ postinjection, mice were anesthetized and immediately perfused with PBS. The brains were removed, weighed, and snap frozen in liquid nitrogen, and then stored at $-80^{\circ} \mathrm{C}$ until processed and analyzed.

Tissue extraction of peptide

Each brain was extracted using acid ethanol. Then, $2.5 \mathrm{ml}$ of acid ethanol per $\mathrm{g}$ of tissue was added, and samples were sonicated, then agitated overnight at $4^{\circ} \mathrm{C}$. Next, samples were centrifuged at $5000 \mathrm{rpm}$ for $30 \mathrm{~min}$ at $4^{\circ} \mathrm{C}$, and the supernatant was poured off. The supernatant was dried for $1 \mathrm{~h} 30 \mathrm{~min}$ in a SpeedVac. The dried samples were reconstituted in the appropriate assay buffer, and necessary dilutions were made for analysis by a fluorescent GLP-1 (active) ELISA kit (Millipore). Values were normalized according to the protein content of samples as estimated by the Bradford method (see also Gengler et al., 2010, for more technical details).

\section{Surgery and electrophysiological recording in the hippocampus area $C A 1$}

The technique used for testing LTP in the hippocampus was exactly as described by Gengler et al. (2010b). Mice were anesthetized with urethane (ethyl carbamate, $1.8 \mathrm{~g} / \mathrm{kg}$, i.p.) for the duration of all experiments. Electrodes (tungsten with Teflon coating; Bilaney) were implanted at coordinates $1.5 \mathrm{~mm}$ posterior and $1.0 \mathrm{~mm}$ lateral for the recording electrode, and for the stimulating electrode, $2.0 \mathrm{~mm}$ posterior to bregma and $1.5 \mathrm{~mm}$ lateral to the midline. The electrodes were lowered through the cortex and the upper layers of the hippocampus and into the CA1 region until the appearance of a negative-deflecting EPSP that had a latency of $\sim 10$ ms. Field EPSPs (fEPSPs) were recorded on a computerized stimulating and recording unit (PowerLab, ADInstruments). The program activated a constant current stimulus isolation unit (NeuroLog System). The high-frequency stimulation protocol for inducing LTP consisted of 3 trains of 100 stimuli at $200 \mathrm{~Hz}$. LTP was measured as percentage of baseline fEPSP ( $n=8$ per group). For paired-pulse facilitation recording, two identical stimuli were given, and the amplitudes of the fEPSPs were compared. The interstimuli interval was increased stepwise to test different facilitating mechanisms (Gengler et al., 2010b).

\section{Histology}

After the LTP studies, animals were perfused transcardially with PBS buffer followed by ice-cold 4\% paraformaldehyde in PBS. Brains were removed and fixed in $4 \%$ paraformaldehyde for at least $24 \mathrm{~h}$ before being transferred to $30 \%$ sucrose solution overnight. Brains were then snap frozen using Envirofreez, and coronal sections of $40 \mu \mathrm{m}$ thickness were cut at a depth of -2 to -3 bregma using a Leica cryostat. Sections were chosen according to stereological rules (Bondolfi et al., 2002), with the first section taken at random and every fifth section afterward. Between 7 and 13 sections were analyzed per brain.

Staining was performed for Ibal (ionized calcium binding adaptor molecule 1), a marker for activated microglia, to measure the inflammation response (Paresce et al., 1997), $\beta$-amyloid plaques, congophilic plaques, synaptophysin, and doublecortin, a marker for immature neurons. All sections were incubated in $3 \% \mathrm{H}_{2} \mathrm{O}_{2}$ to quench endogenous peroxidase activity. For Ibal staining, sections were incubated in $0.05 \mathrm{M}$ trisodium citrate, $\mathrm{pH} 9$, at $90^{\circ} \mathrm{C}$ for $30 \mathrm{~min}$ to enhance antigen recognition. After blocking the sections in 5\% normal serum to avoid nonspecific antibody binding, they were incubated with rabbit polyclonal anti-Ibal (1:2000, Wako Pure Chemical Industries, catalog \#016-20001), goat polyclonal anti-doublecortin (1:200, Santa Cruz Biotechnology, sc-710), or rabbit polyclonal anti-amyloid $\beta$ peptide (1:250, Invitrogen, catalog \#71-5800) 
as shown by Radde et al. (2006), or rabbit antisynaptophysin (1:200, Abcam, catalog \#7837500). After overnight incubation at $4^{\circ} \mathrm{C}$, the sections were incubated in the respective secondary antibodies. For visualization, Vectastain Elite and SG substrate (Vector Laboratories) were used. Congo red staining for congophilic plaques was performed as described by Wilcock et al. (2006). All staining was visualized by Axio Scope 1 (Zeiss) and analyzed using a multithreshold plugin with NIH ImageJ.

ELISA for total soluble $\beta$-amyloid levels Human soluble $\beta$-amyloid oligomer levels were measured using a kit purchased from Invitrogen according to the manufacturer's instructions. Briefly, right hemispheres of control and liraglutide-treated APP/PS1 mouse brains were homogenized in Tris-buffered saline (25 mm Tris- $\mathrm{HCl}, \mathrm{pH} 7.4,150 \mathrm{~mm} \mathrm{NaCl}$ ) supplemented with Protease Inhibitor Cocktail (Sigma, $250 \mu \mathrm{l}$ per $5 \mathrm{ml}$ of buffer). Brain homogenates were centrifuged at $100,000 \times g$ and $4^{\circ} \mathrm{C}$ for $1 \mathrm{~h}$. The supernatant was then diluted 1:10 before carrying out the ELISA, which only measures the soluble $\beta$-amyloid oligomer levels but not amyloid monomers, as detailed in the manufacturer's protocol. Protein was quantified using the Bradford protein assay. The final $\mathrm{A} \beta$ values were determined following normalization to total protein levels ( $n=6$ per group).

\section{ELISA for APP levels}

Human APP levels were measured using a kit purchased from Invitrogen according to the manufacturer's instructions. Briefly, right hemispheres of control and liraglutide-treated APP/PS1 mouse brains were homogenized in cell extraction buffer (Invitrogen) supplemented with Protease Inhibitor Cocktail (Sigma, $250 \mu \mathrm{l}$ per $5 \mathrm{ml}$ of buffer). Brain homogenates were centrifuged at $13,000 \mathrm{rpm}$ and $4^{\circ} \mathrm{C}$ for $10 \mathrm{~min}$. The supernatant was then diluted 1:10 before carrying out the ELISA, as detailed in the manufacturer's protocol. Protein was quantified using the Bradford protein assay. The final APP levels were determined following normalization of total protein levels ( $n=6$ per group).

\section{Statistics}

Data were analyzed using the program Prism (GraphPad); results are expressed as means \pm SEM. Data were analyzed by one-way or two-way ANOVA, followed by post hoc tests, or Student's $t$ tests.

\section{Results}

\section{Effect of acute saline and liraglutide injection on open-field behavior and object recognition}

After a single injection, $30 \mathrm{~min}$ before introduction to the arena, path length and number of line crosses were greater in liraglutidetreated than in saline-treated APP/PS1 animals (Student's $t$ test, $p<0.05, p<0.01$, respectively). Exploratory activity, as shown in number of rearings, increased in liraglutide-treated APP/PS1 and wild-type mice, compared with respective saline controls. Anxiety levels were comparable in all groups. Neither the saline- nor the liraglutide-treated APP/PS1 mice remembered a familiar object after acute administration, while both saline- and liraglutidetreated wild-type mice learned the task well (data not shown).

\section{Object recognition memory is improved after chronic liraglutide administration}

After 8 weeks of treatment with $0.9 \%$ saline or liraglutide ( 25 $\mathrm{nm} / \mathrm{kg}$ bw, i.p.), open-field behavior in APP/PS1 and wild-type mice was similar after chronic drug administration, with explor- atory behavior enhanced in liraglutide-treated APP/PS1 mice (Student's $t$ test, $p=0.0351$ ), and number of line crosses increased in wild-type liraglutide-treated mice (Student's $t$ test, $p=$ 0.043). In both saline- and liraglutide-treated wild-type animals, recognition memory was intact (Fig. $1 A, B$, Student's $t$ test, $p=$ $0.039, p=0.0077$, respectively). Liraglutide-treated APP/PS1 animals' recognition memory was restored (Fig. $1 E$, novel vs familiar, Student's $t$ test, $p=0.0013$ ), while saline-treated APP/PS1 mice failed to discriminate between novel and familiar objects (Fig. $1 D$, Student's $t$ test, $p=0.7964, \mathrm{NS}$ ). A significant increase was observed in the difference score between novel and familiar objects, and between liraglutide- and saline-treated APP/PS1 mice (Fig. $1 F$, Student's $t$ test, $p=0.0495$ ), but not wild-type mice (Fig. 1C).

\section{Spatial memory in the water maze is improved after chronic liraglutide administration}

Escape latency in liraglutide- and saline-treated wild-type mice was comparable in the normal (Fig. 2A) and reversal (Fig. 2B) water maze training paradigms, with escape times reducing across trials, as expected. Escape latency of wild-type liraglutidetreated animals was significantly reduced on day 1 of reversal training (Fig. $2 C$ ), compared with saline-treated wild-type animals (two-way ANOVA, $p<0.01$ ). Both groups spent significantly more time in the target quadrant during the probe trial conducted $24 \mathrm{~h}$ after the last training session in both normal (Fig. $2 B$ ) and reversal (Fig. 2D) trials.

Liraglutide-treated APP/PS1 mice learned the task faster with significantly reduced escape latencies observed on days 3 and 4 of training (Fig. $2 E$, two-way ANOVA; $p<0.001, p<0.01$, respectively) compared with saline-treated controls. Liraglutide-treated mice also remembered the exact location of the platform after the reversal task, while the saline-treated APP/PS1 group did not (Fig. $2 H$, inset; Student's $t$ test, ${ }^{*} p=0.0465$,).

\section{Liraglutide crosses the $\mathrm{BBB}$ to enter the brain}

When measuring total GLP-1 levels in the brain after intraperitoneal injection of $2.5,25$, or $250 \mathrm{nmol} / \mathrm{kg}$ bw liraglutide, a sig- 


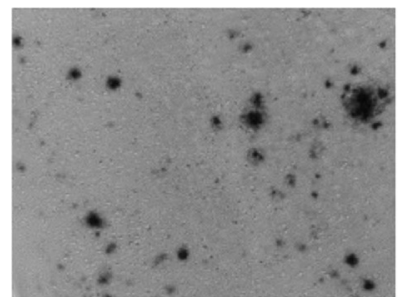

A APP/PS1 Saline

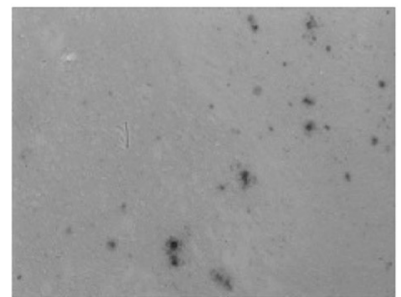

B APP/PS1 + Liraglutide

amyloid plaque load

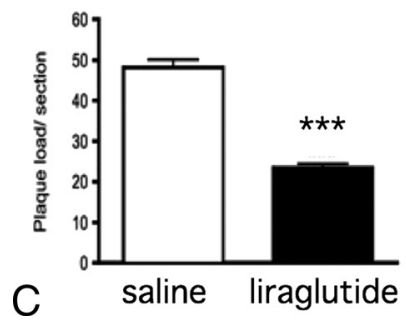

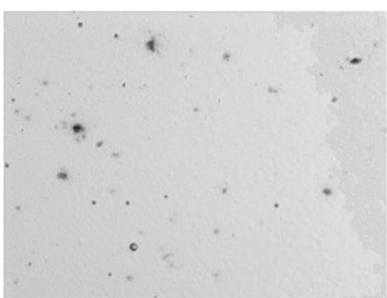

D APP/PS1 Saline

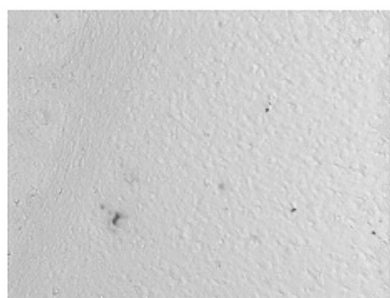

E APP/PS1 + Liraglutide

dense-core plaques

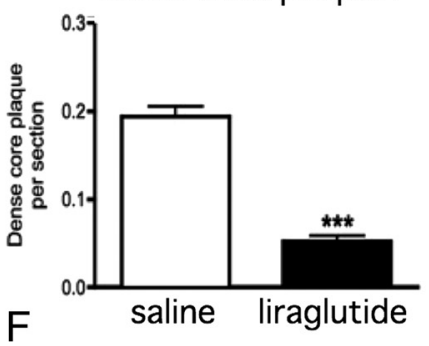

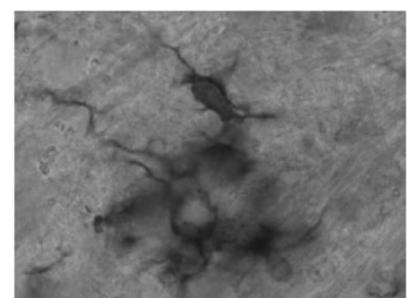

G APP/PS1 Saline

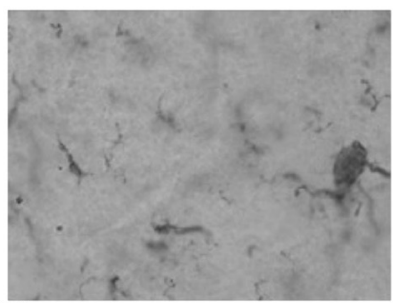

H APP/PS1 + Liraglutide

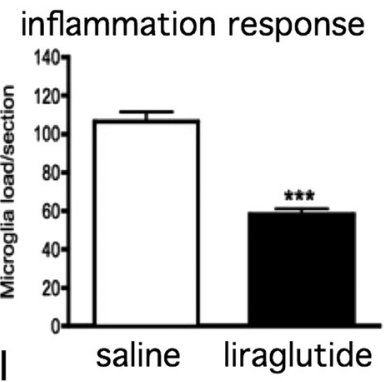

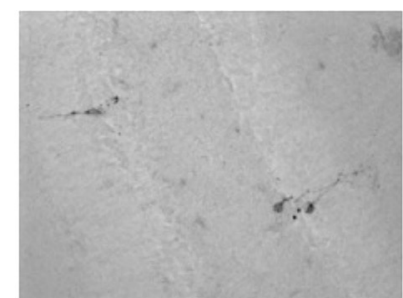
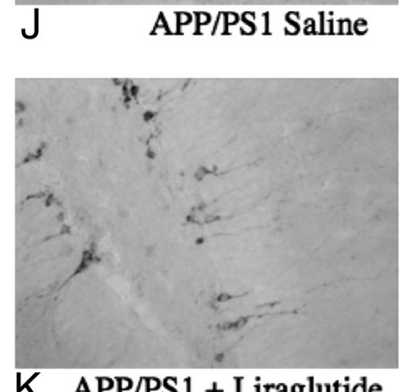

K APP/PS1 + Liraglutide

neurogenesis

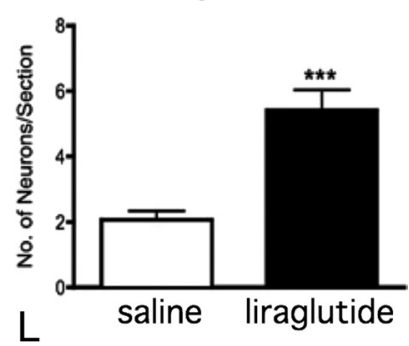

Figure 4. Histological hallmarks of $A D$ are improved with liraglutide. $\boldsymbol{A}-\boldsymbol{C}$, After chronic treatment with $0.9 \%$ saline or liraglutide ( $25 \mathrm{~nm} / \mathrm{kg}$ bw), the number of plaques in the cortex and hippocampus of liraglutide-treated APP/PS1 mice was halved. $\mathbf{D}-\boldsymbol{F}$, The number of Congo red-positive dense core plaques was reduced to $25 \%$. $\mathbf{G}-\mathbf{I}$, The inflammatory response, as shown by activated glia (Iba1 stain), was also halved. $\boldsymbol{J}-\boldsymbol{L}$, Mice treated with liraglutide also had a significant increase in young neurons (doublecortin-positive cells) compared with saline-treated animals. Sample micrographs show saline-treated (top), liraglutide-treated (middle), and overall (bottom) quantification. ${ }^{* * *} p<0.001$ (Student's $t$ test), $n=6$ ).

nificant increase of total GLP-1 signal was found in the ELISA assay $30 \mathrm{~min}$ postinjection for the two higher concentrations. An ANOVA showed an overall significant difference $(p<0.001)$, and post hoc $t$ tests showed the following values (mean \pm SEM): saline control, $195 \pm 17$ pmol; 250 nmol liraglutide, $284 \pm 6$ pmol ( $p<0.01) ; 25$ nmol liraglutide, $247 \pm 13$ pmol $(p<0.05)$; and $2.5 \mathrm{nmol}$ liraglutide, $235 \pm 12 \mathrm{pmol}$ (NS).

\section{Liraglutide enhances induction and maintenance of LTP and PPF in APP/PS1 and wild-type mice}

When testing the inducibility of LTP in the hippocampus in vivo, using a weak stimulation protocol, control APP/PS1 animals were unable to induce LTP, while the liraglutide group showed enhanced LTP (Fig. 3B) (comparing saline and liraglutide, a difference was found between drug group $\mathrm{DF}_{1,1}, F=5.05, p=$ 0.0461; and over time, $\left.\mathrm{DF}_{1,119}, F=2.32, p<0.0001\right)$. Despite having behavior similar to that of saline-treated wild-type mice, liraglutide-treated wild-type mice demonstrated significantly improved LTP (Fig. 3A; drug effect, $F=19.8, \mathrm{DF}_{1,1}, p=0.0010$; change in LTP over time, $\mathrm{DF}_{1,119}, F=2.43, p<0.0001$ ). PPF was also increased in liraglutide-treated APP/PS1 mice at 50, 80, 160, and $200 \mathrm{~ms}$ compared with littermate controls, while no differences were observed in PPF in wild-type animals (Fig. 3C).

\section{Histological hallmarks of Alzheimer's disease are} dramatically reduced in liraglutide-treated APP/PS1 mice $\beta$-Amyloid plaque formation, in cortex, was reduced to $50 \%$ (Fig. $4 C$, Student's $t$ test, $p<0.001)$ in liraglutide-treated APP/PS1 mice (Fig. 4A) compared with saline controls (Fig. 4B). Furthermore, the number of dense-core Congo red plaques was reduced to $25 \%$ (Fig. $4 D-F$, Student's $t$ test, $p<0.001$ ). The inflammatory response, in cortex, as measured by number of activated microglia, was reduced by $50 \%$ in the liraglutide group (Fig. $4 G-I$, Student's $t$ test, $p<0.001$ ). Additionally, the number of young neurons in the dentate gyrus was increased by $65 \%$ (Fig. $4 J-L$, Student's $t$ test, $p<0.001)$.

\section{Synaptophysin levels are increased in liraglutide-treated APP/PS1 mice}

Representative micrographs of synaptophysin staining in both wild-type (Fig. 5A,B) and APP/PS1 (Fig. 5C,D) mice are shown in Figure 5. Quantification of levels of expression of synaptophysin demonstrates that wild-type liraglutide- and saline-treated animals had significantly higher expression levels than both saline- and liraglutide-treated APP/PS1 mice in the polymorph layer of hippocampus (Fig. 5E), molecular layer (Fig. 5G), stratum radiatum (Fig. $5 H$ ), stratum pyramidale (Fig. $5 I$ ), and stratum oriens (Fig. 5J, one-way ANOVA, $p<0.001$ for all regions). Levels in the granular cell layer were also increased in wild-type versus transgenic mice, but to a lesser extent (Fig. $5 F$, one-way ANOVA, $p<0.01$ ). Liraglutide treatment had no effect on synaptophysin levels between wild-type liraglutide- and salinetreated mice, but increased synaptophysin levels in APP/PS1 mice, to varying degrees, in all regions of the hippocampus (Fig. $5 E-J$, one-way ANOVA, $p<0.05$ and $p<0.001$ ), when compared with saline-treated APP/PS1 mice. Liraglutide treatment 


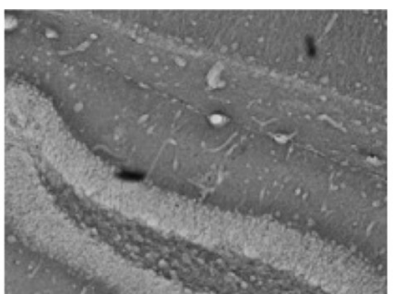

A Wild-Type Saline

$\square$ APP/PS1 Saline

Polymorph layer

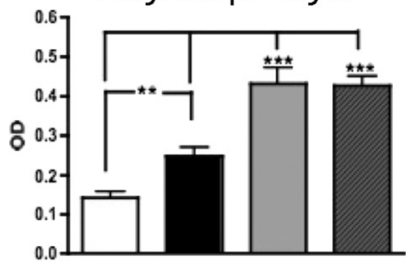

E

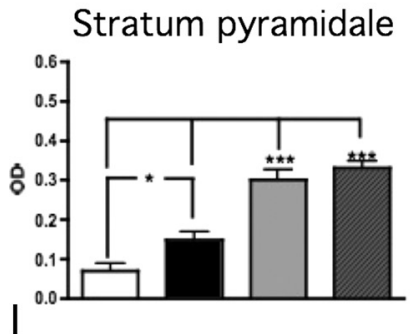

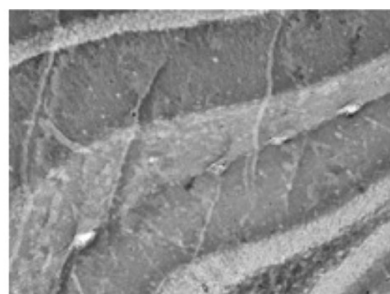

B Wild-type Liraglutide

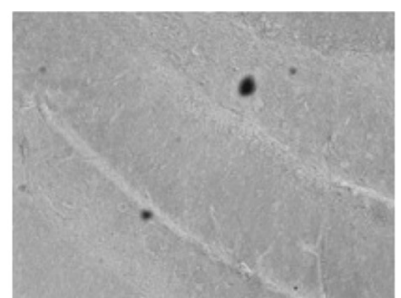

C APP/PS1 Saline

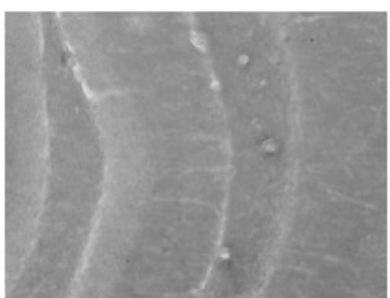

D APP/PS1 Liraglutide

\section{$\square$ APP/PS1 Liraglutide $\square$ Wild-type Saline $\square$ Wild-type Liraglutide}

\section{Hippocampus}

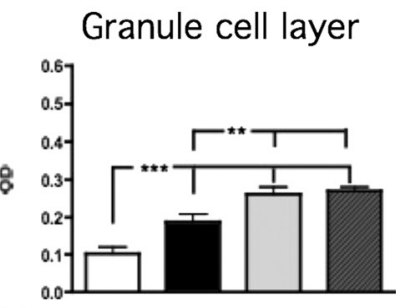

$\mathrm{F}$

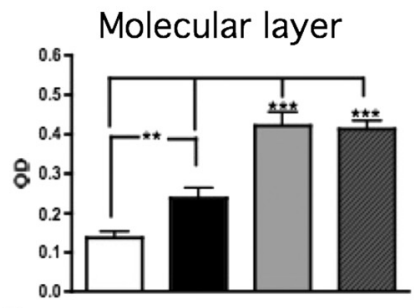

G

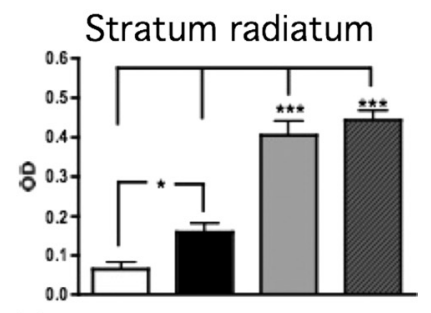

$\mathrm{H}$
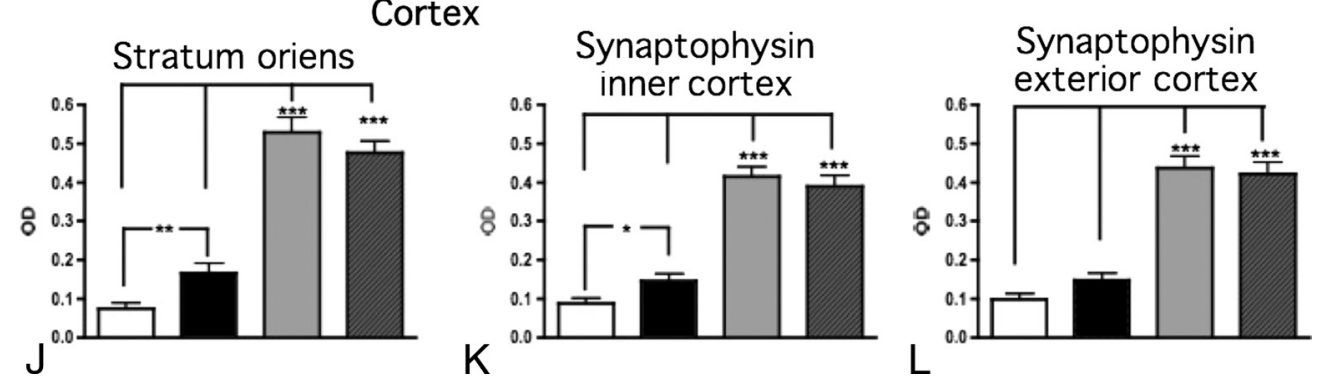

Figure 5. Synaptophysin levels are partially restored by liraglutide treatment in APP/PS1 mice. $\boldsymbol{A}-\boldsymbol{D}$, Representative hippocampal images of synaptophysin-stained brains of both wild-type (A, $\boldsymbol{B})$ and APP/PS1 (C, D ) mice are shown. $\boldsymbol{E}-\boldsymbol{J}$, Quantification of levels of expression of synaptophysin demonstrates that wild-type liraglutide- and saline-treated animals had significantly higher expression levels in all hippocampal regions analyzed than both saline- and liraglutide-treated APP/PS1 mice (one-way ANOVA, $p<0.01$ and $p<0.001$ ). Liraglutide treatment had no effect on synaptophysin levels in wild-type mice, but increased hippocampal synaptophysin levels in APP/PS1 mice, to varying degrees, in all regions (one-way ANOVA, $p<0.05$ and $p<0.001$ ), when compared with saline-treated APP/PS1 mice. $\boldsymbol{K}, \boldsymbol{L}$, Liraglutide treatment also increased synaptophysin levels in the interior $(\boldsymbol{K})$, but not the exterior ( $\boldsymbol{L})$ cortex in liraglutide-treated APP/PS1 mice versus APP/PS1 saline controls. Synaptophysin levels in both exterior and interior cortex were much higher in wild-type mice than in APP/PS1 animals $(p<0.0001)$; however, liraglutide treatment did not change synaptophysin expression in liraglutide-treated wild-type versus saline-treated wild-type mice. ${ }^{*} p<0.05,{ }^{* *} p<0.01,{ }^{* * *} p<0.001, n=6$ per group.

for $56 \mathrm{~d}$ increased synaptophysin levels in the interior (Fig. $5 \mathrm{~K}$, one-way ANOVA, $p<0.05$ ), but not the exterior cortex (Fig. $5 L$ ) in liraglutide-treated APP/PS1 mice versus APP/PS1 saline controls. Synaptophysin levels in both exterior and interior cortex were much greater in wild-type mice than in APP/PS1 animals (Fig. $5 K, L$, one-way ANOVA, $p<0.0001$ ); however, liraglutide treatment did not change synaptophysin expression in liraglutide-treated wild-type versus saline-treated wild-type mice (Fig. $5 K, L, n=6$ per group).

\section{Soluble A $\beta$ oligomer and total APP levels are significantly} reduced by liraglutide treatment

Liraglutide treatment significantly reduced aggregated $\beta$-amyloid levels compared with saline-treated controls, $0.13 \pm 0.0049$ versus $0.0995 \pm 0.0105 \mathrm{ng} / \mathrm{mg}$ protein (Fig. $6 \mathrm{~A}$, Student's $t$ test, $p=0.0109)$. Brain APP levels were also significantly reduced by liraglutide treatment, $21.13 \pm 3.59$ versus $11.67 \pm 1.754 \mathrm{ng} / \mathrm{mg}$ protein (Fig. $6 B$, Student's $t$ test, $p=0.0272$ ).

\section{Discussion}

This study demonstrates that liraglutide, a drug that is on the market as a treatment for T2DM, has a range of beneficial pro- tective properties in an APP/PS1 mouse model of AD not previously encountered. The GLP-1 receptor agonist crosses the BBB and can prevent the deterioration of learning in object recognition and water maze tasks. The levels of performance in the drugtreated group are on the same level as in wild-type controls. These behavioral improvements were supported by a significant protection of LTP and paired-pulse facilitation in the liraglutide-treated APP/PS1 mice. The reduction of synaptic numbers in this APP/ PS1 mouse model was partly prevented by liraglutide. This indicates that synaptic number and function are protected, which also prevents cognitive impairments. Impressively, the overall number of plaques in the cortex of the mice was halved, and the number of dense-core Congo red-positive plaques was reduced even further. Also, the amount of soluble amyloid oligomers was reduced. Aggregated amyloid species are considered to be toxic and more detrimental to neuronal development than plaques (Townsend et al., 2006). As plaque load and soluble oligomer levels were reduced, we analyzed the levels of the amyloid precursor protein in the brain to identify whether the reduction is the result of reduced synthesis or of increased clearance of amyloid. We found that APP levels were indeed reduced in the brain, 
A Soluble amyloid oligomer levels
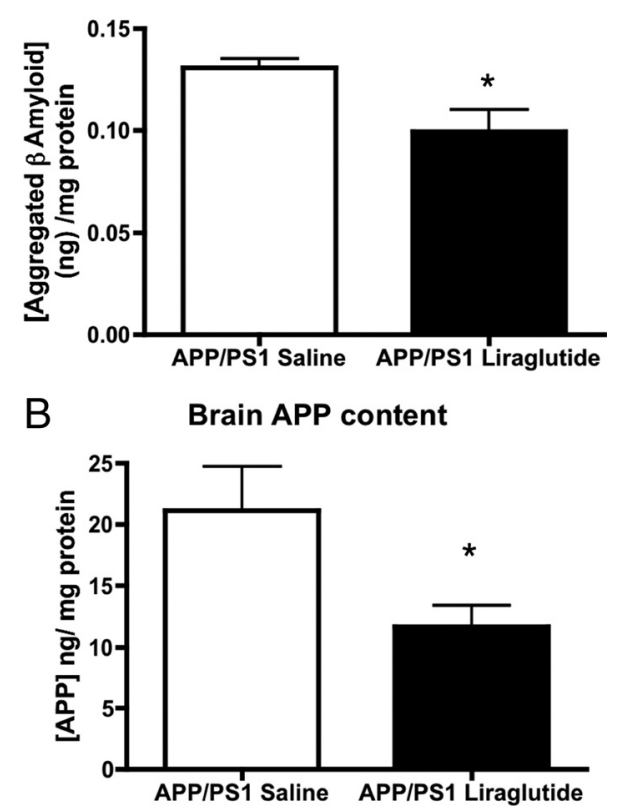

Figure 6. Soluble A $\beta$ oligomer and APP levels are reduced in liraglutide-treated APP/PS1 mice. Oligomer $\beta$-amyloid levels and APP levels were measured by ELISA. Liraglutide significantly reduced both oligomer $\beta$-amyloid levels $(\boldsymbol{A})$ (Student's test, $p=0.0109$ ) and brain APP levels $(\boldsymbol{B})$ (Student's $t$ test, $p=0.0272$ ).

showing that synthesis of amyloid is most likely reduced. However, this does not exclude the possibility that clearance of amyloid is also increased. Further studies will be needed to address this aspect. Importantly, the inflammation response in the brain was much reduced, as evaluated by the number of activated microglia. Additionally, the number of new neurons in the dentate gyrus was normalized to wild-type control levels in the liraglutide-treated APP/PS1 mice. This confirms previous findings that show that peripheral injection of liraglutide increases neuronal progenitor cell proliferation and neurogenesis in the brain (Hamilton et al., 2011). The fact that neuroneogenesis is normalized in these mice is most interesting. While it is not completely understood what role the replacement of neurons plays in the brain, and whether or not these neurons are actually functionally integrated into the cortical networks, it has been suggested that the activation of stem cells and the replacement of lost neurons may be of benefit and could potentially repair some of the damage caused by AD (Korecka et al., 2007).

The results confirm previous findings testing the GLP-1 analog Val(8)GLP-1, which showed a protection of LTP and PPF in the hippocampus in 9- and 18-month-old APP/PS1-21 mice after 3 weeks of chronic intraperitoneal injection. However, the total plaque load and the inflammation response were not changed (Gengler et al., 2010b). These differences may be attributable to the increased duration of injection in the current study and the difference in age at commencement of study.

Clearly, the removal of plaques and the reduction of amyloid levels in the brain is a very desirable result. Furthermore, the reduction of the inflammation response is an important aspect in this context. Inflammation and the detrimental long-term effects are one of the important underlying mechanisms of neurodegeneration found in $\mathrm{AD}$ (Aisen, 2002). In vitro studies have shown that GLP-1 reduces cytokine release after stimulation with lipopolysaccharide (Iwai et al., 2006), suggesting that GLP-1 directly reduces cytokine release. However, it may be that the reduced cell count in activated microglia in the present study is caused by the reduction of plaques in the brain, which also reduces the inflammation response. Further research will have to investigate what the exact action of liraglutide is.

What could be the basis for the improvements of LTP and memory formation? Synaptic numbers in the brains of APP/PS1 mice are reduced, as they are in the brains of $\mathrm{AD}$ (LaFerla and Oddo, 2005; Radde et al., 2006). This loss can explain cognitive impairment and reductions in LTP and paired-pulse facilitation. Since the reduction in numbers of synapses is less in the liraglutide-treated APP/PS1 mice, it seems that liraglutide protects synapses or induces synaptogenesis, and prevents cognitive impairment and loss of LTP and paired-pulse facilitation.

The described effects on cognition, synapse formation, and LTP mirror other studies that demonstrate the neuroprotective effects of growth factors in mouse models of $\mathrm{AD}$. In one study, brain-derived neurotrophic factor (BDNF) injected intracerebroventricularly led to an enhancement in hippocampal synaptic density (Blurton-Jones et al., 2009), while gene delivery of BDNF in rodents and primates improved cognition and synaptic signaling (Nagahara et al., 2009), without altering plaque load. Growth factors also enhance stem cell division and cellular repair processes (Holscher, 2010). It is therefore to be assumed that GLP-1 receptors activate a similar growth factor-like signaling cascade (see Holscher, 2010, for a detailed discussion). Liraglutide treatment has many advantages over these and other growth factors, given the level of reduction of $\beta$-amyloid, dense Congo red-positive plaques, and inflammation, and the increase in neurogenesis observed in the current study. Another major disadvantage of these growth factors is that they do not cross the $\mathrm{BBB}$ and need to be injected intracerebroventricularly or delivered by gene delivery systems, which are not suitable for the treatment of large numbers of patients. A different growth factor that has shown great promise as a treatment for neurodegenerative disorders is nerve growth factor (NGF). Again, NGF was found to protect synapses, LTP, and learning abilities in $\mathrm{AD}$ mouse models and non-primate monkeys, though amyloid plaque load was not affected (Clarris et al., 1994; Kordower et al., 1997; Bradbury, 2005; Covaceuszach et al., 2009). However, NGF does not cross the $\mathrm{BBB}$ either, and therefore, gene delivery systems have been developed to be able to use NGF as a treatment of CNS disorders. Such attempts to increase the amount of NGF production in the CNS have not been successful so far (Clarris et al., 1994; Kordower et al., 1997; Bradbury, 2005; Heese et al., 2006; SchulteHerbrüggen et al., 2007).

In comparison, liraglutide can be injected peripherally once daily and can be taken by nondiabetic people (Vella et al., 2002). It is already on the market as a T2DM treatment (Victoza) and is well tolerated. If the improvements in memory and synaptic plasticity, reduction in neurotoxic amyloid oligomer levels, numbers of amyloid plaques, and inflammation response, and increased neurogenesis translate to humans, GLP-1 analogs such as liraglutide are a promising new treatment for Alzheimer's disease. Clinical trials would be needed to verify the effects in humans.

\section{References}

Abbas T, Faivre E, Hölscher C (2009) Impairment of synaptic plasticity and memory formation in GLP-1 receptor KO mice: interaction between type 2 diabetes and Alzheimer's disease. Behav Brain Res 205:265-271.

Aisen PS (2002) The potential of anti-inflammatory drugs for the treatment of Alzheimer's disease. Lancet Neurol 1:279-284.

Blurton-Jones M, Kitazawa M, Martinez-Coria H, Castello NA, Müller FJ, Loring JF, Yamasaki TR, Poon WW, Green KN, LaFerla FM (2009) 
Neural stem cells improve cognition via BDNF in a transgenic model of Alzheimer disease. Proc Natl Acad Sci U S A 106:13594-13599.

Bondolfi L, Calhoun M, Ermini F, Kuhn HG, Wiederhold KH, Walker L, Staufenbiel M, Jucker M (2002) Amyloid-associated neuron loss and gliogenesis in the neocortex of amyloid precursor protein transgenic mice. J Neurosci 22:515-522.

Bradbury J (2005) Hope for AD with NGF gene-therapy trial. Lancet Neurol 4:335.

Clarris HJ, Nurcombe V, Small DH, Beyreuther K, Masters CL (1994) Secretion of nerve growth factor from septum stimulates neurite outgrowth and release of the amyloid protein precursor of Alzheimer's disease from hippocampal explants. J Neurosci Res 38:248-258.

Covaceuszach S, Capsoni S, Ugolini G, Spirito F, Vignone D, Cattaneo A (2009) Development of a non invasive NGF-based therapy for Alzheimer's disease. Curr Alzheimer Res 6:158-170.

Craft S (2007) Insulin resistance and Alzheimer's disease pathogenesis: potential mechanisms and implications for treatment. Curr Alzheimer Res 4:147-152.

During MJ, Cao L, Zuzga DS, Francis JS, Fitzsimons HL, Jiao X, Bland RJ, Klugmann M, Banks WA, Drucker DJ, Haile CN (2003) Glucagon-like peptide- 1 receptor is involved in learning and neuroprotection. Nat Med 9:1173-1179.

Gengler S, Hamilton A, Hölscher C (2010a) Synaptic plasticity in the hippocampus of a APP/PS1 mouse model of Alzheimer's disease is impaired in old but not young mice. PLoS One 5:e9764.

Gengler S, McClean P, McCurtin R, Gault V, Holscher C (2010b) $\mathrm{Val}(8)$ GLP-1 rescues synaptic plasticity and reduces dense core plaques in APP/PS1 mice. Neurobiol Aging. Advance online publication. Retrieved March 10, 2011. doi:10.1016/j.neurobiolaging.2010.1002.1014.

Greenberg DA, Jin K (2006) Neurodegeneration and neurogenesis: focus on Alzheimer's disease. Curr Alzheimer Res 3:25-28.

Hamilton A, Patterson S, Porter D, Gault VA, Holscher C (2011) Novel GLP-1 mimetics developed to treat type 2 diabetes promote progenitor cell proliferation in the brain. J Neurosci Res 89:481-489.

Heese K, Low JW, Inoue N (2006) Nerve growth factor, neural stem cells and Alzheimer's disease. Neurosignals 15:1-12.

Hölscher C (2005) Development of beta-amyloid-induced neurodegeneration in Alzheimer's disease and novel neuroprotective strategies. Rev Neurosci 16:181-212.

Holscher C (2010) Incretin Analogues that have been Developed to Treat Type 2 Diabetes Hold Promise as a Novel Treatment Strategy for Alzheimer's Disease. Recent Pat CNS Drug Discov 5:109-117.

Hölscher C, Li L (2010) New roles for insulin-like hormones in neuronal signalling and protection: New hopes for novel treatments of Alzheimer's disease? Neurobiol Aging 31:1495-1502.

Hoyer S (2004) Glucose metabolism and insulin receptor signal transduction in Alzheimer disease. Eur J Pharmacol 490:115-125.

Iwai T, Ito S, Tanimitsu K, Udagawa S, Oka J (2006) Glucagon-like peptide-1 inhibits LPS-induced IL-1beta production in cultured rat astrocytes. Neurosci Res 55:352-360.
Jankowsky JL, Slunt HH, Ratovitski T, Jenkins NA, Copeland NG, Borchelt DR (2001) Co-expression of multiple transgenes in mouse CNS: a comparison of strategies. Biomol Eng 17:157-165.

Kastin AJ, Akerstrom V, Pan W (2002) Interactions of glucagon-like peptide-1 (GLP-1) with the blood-brain barrier. J Mol Neurosci 18:7-14.

Kordower JH, Mufson EJ, Fox N, Martel L, Emerich DF (1997) Cellular delivery of NGF does not alter the expression of beta-amyloid immunoreactivity in young or aged nonhuman primates. Exp Neurol 145:586-591.

Korecka JA, Verhaagen J, Hol EM (2007) Cell-replacement and genetherapy strategies for Parkinson's and Alzheimer's disease. Regen Med 2:425-446

LaFerla FM, Oddo S (2005) Alzheimer's disease: Abeta, tau and synaptic dysfunction. Trends Mol Med 11:170-176.

Lovshin JA, Drucker DJ (2009) Incretin-based therapies for type 2 diabetes mellitus. Nat Rev Endocrinol 5:262-269.

Nagahara AH, Merrill DA, Coppola G, Tsukada S, Schroeder BE, Shaked GM, Wang L, Blesch A, Kim A, Conner JM, Rockenstein E, Chao MV, Koo EH, Geschwind D, Masliah E, Chiba AA, Tuszynski MH (2009) Neuroprotective effects of brain-derived neurotrophic factor in rodent and primate models of Alzheimer's disease. Nat Med 15:331-337.

Paresce DM, Chung H, Maxfield FR (1997) Slow degradation of aggregates of the Alzheimer's disease amyloid beta-protein by microglial cells. J Biol Chem 272:29390-29397.

Perry T, Lahiri DK, Sambamurti K, Chen D, Mattson MP, Egan JM, Greig NH (2003) Glucagon-like peptide-1 decreases endogenous amyloid-beta peptide (Abeta) levels and protects hippocampal neurons from death induced by Abeta and iron. J Neurosci Res 72:603-612.

Perry T, Holloway HW, Weerasuriya A, Mouton PR, Duffy K, Mattison JA, Greig NH (2007) Evidence of GLP-1-mediated neuroprotection in an animal model of pyridoxine-induced peripheral sensory neuropathy. Exp Neurol 203:293-301.

Radde R, Bolmont T, Kaeser SA, Coomaraswamy J, Lindau D, Stoltze L, Calhoun ME, Jäggi F, Wolburg H, Gengler S, Haass C, Ghetti B, Czech C, Hölscher C, Mathews PM, Jucker M (2006) Abeta42-driven cerebral amyloidosis in transgenic mice reveals early and robust pathology. EMBO Rep 7:940-946.

Schulte-Herbrüggen O, Braun A, Rochlitzer S, Jockers-Scherübl MC, Hellweg R (2007) Neurotrophic factors - a tool for therapeutic strategies in neurological, neuropsychiatric and neuroimmunological diseases? Curr Med Chem 14:2318-2329.

Townsend M, Shankar GM, Mehta T, Walsh DM, Selkoe DJ (2006) Effects of secreted oligomers of amyloid beta-protein on hippocampal synaptic plasticity: a potent role for trimers. J Physiol 572:477-492.

Vella A, Shah P, Reed AS, Adkins AS, Basu R, Rizza RA (2002) Lack of effect of exendin-4 and glucagon-like peptide-1-(7,36)-amide on insulin action in non-diabetic humans. Diabetologia 45:1410-1415.

Wilcock DM, Gordon MN, Morgan D (2006) Quantification of cerebral amyloid angiopathy and parenchymal amyloid plaques with Congo red histochemical stain. Nat Protoc 1:1591-1595. 